\title{
Association of forkhead box J3 (FOXJ3) polymorphisms with rheumatoid arthritis
}

\author{
JU YEON BAN ${ }^{1 *}$, HAE JEONG PARK ${ }^{2 *}$, SU KANG KIM ${ }^{2}$, JONG WOO KIM ${ }^{2}$,

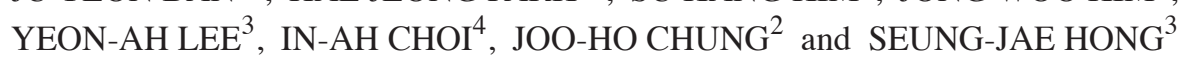

\author{
${ }^{1}$ College of Dentistry and Institute of Tissue Regeneration Engineering, Dankook University, Cheonan 330714; \\ ${ }^{2}$ Kohwang Medical Research Institute; ${ }^{3}$ Division of Rheumatology, Department of Internal Medicine, School of Medicine, \\ Kyung Hee University, Seoul 130702; ${ }^{4}$ Division of Rheumatology, Department of Internal Medicine, \\ School of Medicine, Chung-Buk National University, Cheongju 361711, Republic of Korea
}

Received March 21, 2013; Accepted July 23, 2013

DOI: $10.3892 / \mathrm{mmr} .2013 .1623$

\begin{abstract}
The aim of this study was to investigate the correlation between single nucleotide polymorphisms (SNPs) of the forkhead box J3 (FOXJ3) gene and rheumatoid arthritis (RA). For the association studies, 307 patients with RA and 476 control patients without RA were recruited. Eleven SNPs (rs2282404, rs2455084, rs1393009, rs7539485, rs4660616, rs510157, rs343386, rs12732892, rs343389, rs343376 and rs585320) were genotyped using direct sequencing and the resulting data were analyzed using the SNPStats, Haploview and Helixtree programs. Seven SNPs (rs2455084, rs1393009, rs7539485, rs4660616, rs510157, rs343386 and rs343389) were associated with RA in three alternative models (log-additive, dominant and recessive models; $\mathrm{P}<0.05)$. A strong linkage disequilibrium block, including all 11 SNPs, was constructed using the Gabriel method. Two haplotypes, TCCTTGTCTTT and TCTTCTGTCAC, were significantly associated with RA $(\mathrm{P}<0.05)$. In clinical characteristic analysis, the SNP rs585320 was also associated with the anti-cyclic citrullinated peptide. These results suggest that FOXJ3 may be associated with the development of RA.
\end{abstract}

\section{Introduction}

Rheumatoid arthritis (RA) is an autoimmune disease that affects $\sim 1 \%$ of the adult population (1). RA is considered to be a complex disease and its etiology and pathogenesis remain unclear (2). Genetic and environmental factors, and their interactions, are

Correspondence to: Dr Seung-Jae Hong, Division of Rheumatology, Department of Internal Medicine, School of Medicine, Kyung Hee University, Hoegi-dong, Dongdaemun-gu, Seoul 130702, Republic of Korea

E-mail: hsj718@paran.com

"Contributed equally

Key words: forkhead box J3, haplotype, linkage disequilibrium, polymorphism, rheumatoid arthritis involved in the development of RA (3). The strongest genetic associations are with genes located in the human leukocyte antigen (HLA) region, particularly the HLA-DRB1 gene $(4,5)$. Rheumatoid factor (RF) production in RA patients is correlated with smoking. Mattey et al (6) demonstrated that patients that were HLA-DRB1*0401 positive and had previously smoked were likely to be RF positive. Various genes, including the protein tyrosine phosphatase non-receptor 22 (PTPN22) gene, are also known to be associated with RA (7). Karlson et al (8) demonstrated that HLA-DRB1 shared epitope (HLA-SE) and PTPN22 were correlated with presence of cyclic citrullinated peptide (CCP) and RF. However, the strength of the association between these genes and RA vary geographically. For example, PTPN22 is associated with RA in populations of European origin, but not in non-European populations $(9,10)$. In contrast, peptidyl arginine deiminase type IV, solute carrier family 22 (organic cation/ergothioneine transporter) member 4 and Fc receptor-like 3 are associated with RA in the Japanese population and in other Asian groups (11-13); however, yields weak or negative results in European populations $(14,15)$. These divergent results suggest the genetic heterogeneity of RA across human populations.

Forkhead proteins are a large family of functionally diverse transcription factors that have been implicated in a variety of cellular processes (16). Forkhead box J3 (FOXJ3) is a member of the FOX gene family, which encodes transcription factors composed of a conserved DNA binding motif termed the forkhead domain (17). Based on interpretations of the mouse and human genome sequences, human FOX genes have $\sim 43$ members, which have been classified into 17 subclasses: FOXA to FOXQ (18). Numerous members of this family are crucial in the regulation of immune responses and it has been demonstrated that FOXP3 is important in autoimmune diseases (19). Li et al (20) showed that a FOXJ1 polymorphism (rs3192453, 3375G $>$ C) was associated with allergic rhinitis and that another FOXJ1 polymorphism was related to systemic lupus erythematosus and RA in the Korean population (21). However, no genetic study of FOXJ3 has currently been conducted.

In the present study, it was investigated whether FOXJ3 polymorphisms were correlated with susceptibility to RA. 
Table I. Primer sequences for FOXJ3 polymorphisms.

\begin{tabular}{|c|c|c|}
\hline SNP & Primer & Size (bp) \\
\hline rs2282404 & $\begin{array}{l}\text { Sense: 5'-CCATAACCTGGGTAGCTACCTTGAG-3' } \\
\text { Antisense: 5'-TTCCGAGCGACTTGGGGTCCTTGT-3' }\end{array}$ & 441 \\
\hline rs 2455084 & $\begin{array}{l}\text { Sense: 5'-TGCGATGGCTGCTTCCTAAATGTGG-3' } \\
\text { Antisense: 5'-AAATGACGTCCTGAACCCTAGCCTT-3' }\end{array}$ & 488 \\
\hline rs1393009 & $\begin{array}{l}\text { Sense: 5'-GAACCTTGCATGCTTAACTCTGTGG-3' } \\
\text { Antisense: 5'-ACAAGCCTGTCCATACAAACCCATC-3' }\end{array}$ & 452 \\
\hline rs7539485 & $\begin{array}{l}\text { Sense: 5'-CATGATCATAGCTCACTGCAGCCT-3' } \\
\text { Antisense: 5'-CATCAGACAAGTCAGTGGGGAAAGG-3' }\end{array}$ & 603 \\
\hline rs4660616 & $\begin{array}{l}\text { Sense: 5'-CTGGCTGACTCAGTATGAGAAGTGA-3' } \\
\text { Antisense: 5'-GGAAGATCTGCCATGTGCCCTAAA-3' }\end{array}$ & 492 \\
\hline rs510157 & $\begin{array}{l}\text { Sense: 5'-CCTGGCCACAAAGCGAGGTTTAAA-3' } \\
\text { Antisense: 5'-TGTAATTCCAGGTGATTTGGGGGGT-3' }\end{array}$ & 557 \\
\hline rs343386 & $\begin{array}{l}\text { Sense: 5'-GAGCTTCCCTTTGGTCCTTCTTGTG-3' } \\
\text { Antisense: 5'-AGACAGCTACATCATTCGGGAGCT-3' }\end{array}$ & 757 \\
\hline rs12732892 & $\begin{array}{l}\text { Sense: 5'-GATTAGTGTGGCTGTCAGTGTATTG-3' } \\
\text { Antisense: 5'-CATACCAAAACCTATGGAGTATAGC-3' }\end{array}$ & 512 \\
\hline rs343389 & $\begin{array}{l}\text { Sense: 5'-CCATTCAGGTAGTCTGTACCTTT-3' } \\
\text { Antisense: 5'-GCAGAAACCTTACAGACCAGGAGAG-3' }\end{array}$ & 481 \\
\hline rs343376 & $\begin{array}{l}\text { Sense: 5'-CCATGTAGATTTGTGAGGTCCTGGG-3' } \\
\text { Antisense: 5'-CCTAAACTGTCCCTCAGAGTTAAG-3' }\end{array}$ & 661 \\
\hline rs585320 & $\begin{array}{l}\text { Sense: 5'-GTGTCAACATGGTTTCCAGCTGTAG-3' } \\
\text { Antisense: 5'-CTGCTACGTTTTTCTTTCCCGTGTG-3' }\end{array}$ & 433 \\
\hline
\end{tabular}

FOXJ3, forkhead box J3; SNP, single nucleotide polymorphism; bp, base pair.

\section{Patients and methods}

Patients. A case-control study was conducted to investigate the correlation between RA and FOXJ3 polymorphisms. Unrelated patients $(n=307)$ with RA (59 males and 248 females) were recruited from rheumatology centers in the Republic of Korea (Kyung Hee University Hospital, Seoul, Korea). Each patient was diagnosed by a rheumatologist based on the ACR 1987 rheumatoid arthritis diagnostic criteria (22). For the control group, 476 subjects (207 males and 269 females) were recruited from participants undergoing a general health check-up program, following the confirmation of no clinical implication of rheumatic disease or any other disorder. All procedures were conducted according to the Declaration of Helsinki guidelines. Written informed consent was obtained from each subject. This study was approved by the Ethics Review Committee of the Medical Research Institute, School of Medicine, Kyung Hee University (Seoul, Korea).

Clinical data, such as gender, age at disease onset and the presence of bone erosion were obtained by reviewing medical records at the time of enrollment. Laboratory data included erythrocyte sedimentation rate (ESR), C-reactive protein (CRP), anti-cyclic citrullinated peptide (anti-CCP) and RF values.

SNP selection and genotyping. All tagging SNPs of FOXJ3 analyzed in this study were selected from our unpublished results of a $100 \mathrm{~K}$ genome-wide association analysis (Illumina, Inc., San Diego, CA, USA) in RA. From the International HapMap Project data set (http://www.hapmap.org), the SNPs with an optimal minor allele frequency $(\geq 5 \%)$, heterozygosity $>0.1$ and the best coverage to serve as tag SNPs were genotyped. A total of 11 SNPs [rs2282404 (5'-near gene), rs2455084 (intron 1), rs1393009 (intron 1), rs7539485 (intron 2), rs4660616 (intron 3), rs510157 (intron 4), rs343386 (intron 4), rs12732892 (intron 4), rs343389 (intron 4), rs343376 (exon 5, Ala161Val) and rs585320 (intron 7)] were selected. The locations of the 11 selected SNPs in the region of FOXJ3 are shown in Fig. 1A. Genomic DNA was extracted using a commercially available Qiagen DNA extraction kit (Qiagen, Tokyo, Japan) from blood samples collected in EDTA. SNP genotyping was performed using direct sequencing with genomic DNA that was amplified using primers for each SNP. The sequences of the primers are shown in Table I. The samples were sequenced using an ABI Prism 377 automatic sequencer (Applied Biosystems, Inc., Foster City, CA, USA). Sequence data were analyzed using the SeqManII software (DNAStar, Inc., Madison, WI, USA).

Statistical analysis. Hardy-Weinberg equilibrium (HWE) for 11 SNPs was assessed using SNPStats (http://bioinfo.iconcologia.net/SNPstats). A linkage disequilibrium (LD) block of polymorphisms was tested using Haploview version 4.2 (Broad Institute of MIT and Harvard, Cambridge, MA, USA). The haplotypes and their frequencies were calculated by the expectation-maximization algorithm. Multiple logistic regression analyses were conducted to calculate the odds ratios, 95\% confidence intervals and corresponding P-values. SNPStats, HapAnalyzer, Helixtree software (Golden Helix, Inc., Bozeman, MT, USA) and SNPAnalyzer (ISTECH, Inc., Goyang, Korea) were used to evaluate genetic data. Logistic regression analysis in all three analysis models (log-additive, dominant and recessive models for rare alleles) was utilized to demonstrate alternative effects of the variants (23). 
Table II. General characteristics of study subjects.

Age (years, mean $\pm \mathrm{SD})$

$46.56 \pm 12.91$

$59 / 248$

$44.45 \pm 12.70$

Gender (male/female)

Disease duration (years, mean $\pm \mathrm{SD}$ )

Erythrocyte sedimentation rate $(\mathrm{mm} / \mathrm{h}$, mean $\pm \mathrm{SD})$

C-reactive protein $(\mathrm{mg} / \mathrm{dl}$, mean $\pm \mathrm{SD})$

Patients with bone erosion ( $\mathrm{n}$ )

$4.68 \pm 4.53$

$41.00 \pm 29.29$

$2.26 \pm 4.84$

143

268

RF positive ( $\mathrm{n}$ )

Anti-CCP positive (n)
$207 / 269$

RA patients $(\mathrm{n}=130)$ which were not analyzed for the presence of anti-CCP were excluded. RA, rheumatoid arthritis; $\mathrm{n}$, number of subjects; RF, rheumatoid factor; anti-CCP, anti-cyclic citrullinated peptide.

A

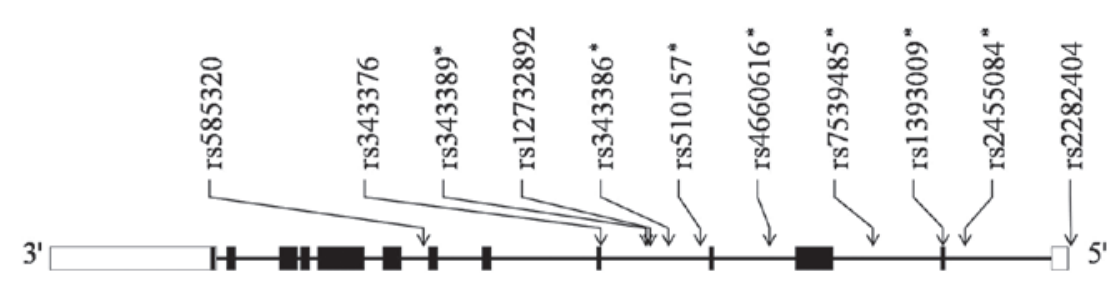

B
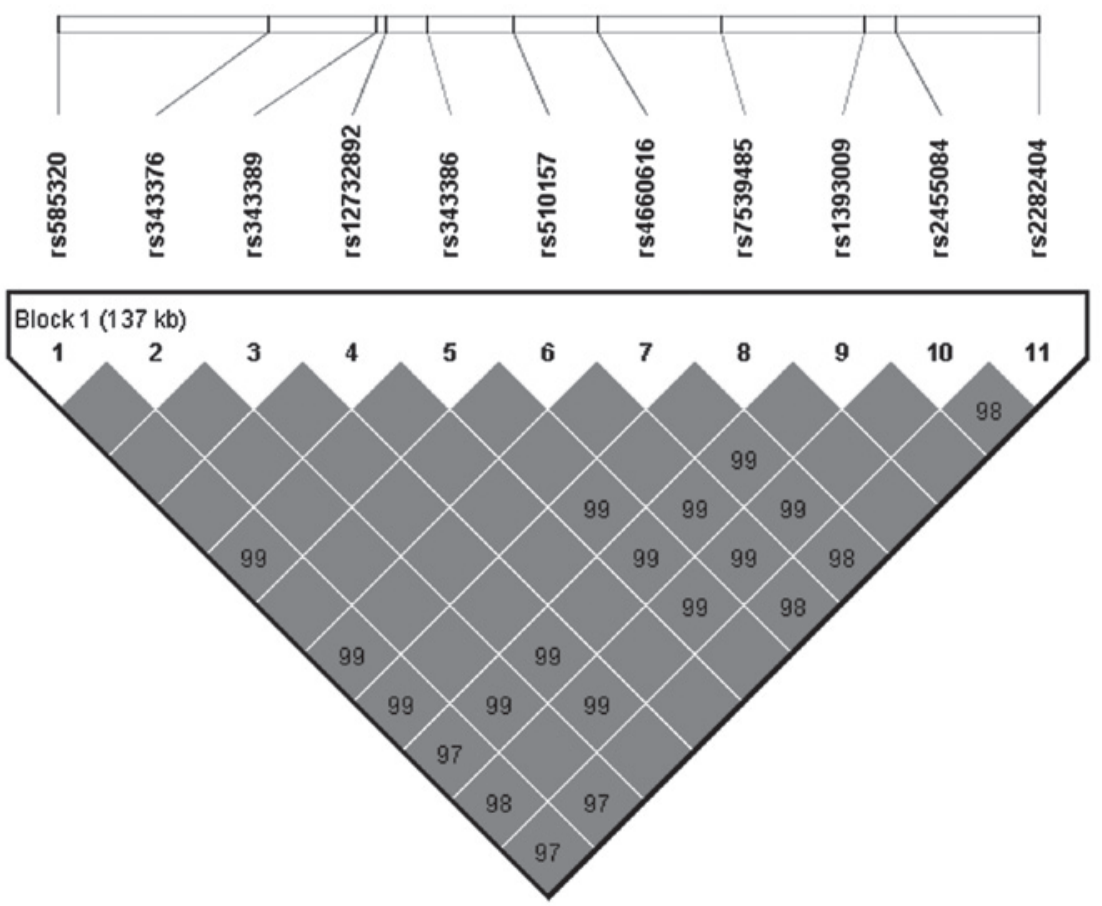

Figure 1. Gene map and LD in FOXJ3. (A) Gene map of SNPs in FOXJ3 on chromosome 1p34.2. Exons are boxed, coding regions are represented by black boxes and untranslated regions by white boxes. "Indicates significant SNPs. Arrows indicate the location of each SNP. (B) LD coefficient (|D'|) and an LD block among FOXJ3 SNPs visualized using Haploview 4.2. LD, linkage disequilibrium; FOXJ3, forkhead box J3; SNPs, single nucleotide polymorphisms.

$\mathrm{P}<0.05$ was considered to indicate a statistically significant difference.

\section{Results}

The clinical characteristics of the 307 RA patients (248 females and 59 males) and 476 control subjects are shown in Table II. The mean age of the RA patients was $45.56 \pm 12.91$ years and the mean age of the control subjects was $44.45 \pm 12.70$ years.
The mean disease duration was $4.68 \pm 4.53$ years. The mean level of ESR in RA patients was $41.00 \pm 29.29 \mathrm{~mm} / \mathrm{h}$ and CRP was $2.26 \pm 4.84 \mathrm{mg} / \mathrm{dl}$. Among the $307 \mathrm{RA}$ patients, 268 patients were positive for RF (87.3\%) and 143 patients exhibited bone erosion $(46.6 \%)$.

In the control group, the genotype distributions for all SNPs were in HWE (data not shown). The genotype frequencies of the polymorphisms were compared between the RA and the normal control groups using logistic regression models with 
Table III. Genotype frequencies of FOXJ3 polymorphisms in patients with rheumatoid arthritis and control subjects.

\begin{tabular}{|c|c|c|c|c|c|c|c|c|c|}
\hline \multirow{2}{*}{$\frac{\text { SNP }}{\text { (Locus) }}$} & \multirow[b]{2}{*}{ Genotype } & \multirow{2}{*}{$\frac{\mathrm{RA}}{\mathrm{n}(\%)}$} & \multirow{2}{*}{$\begin{array}{c}\text { Control } \\
\mathrm{n}(\%)\end{array}$} & \multicolumn{2}{|c|}{ Log-additive } & \multicolumn{2}{|c|}{ Dominant } & \multicolumn{2}{|c|}{ Recessive } \\
\hline & & & & OR $(95 \%$ CI $)$ & P-value & OR $(95 \%$ CI $)$ & P-value & OR $(95 \% \mathrm{CI})$ & P-value \\
\hline $\begin{array}{l}\text { rs2282404 } \\
\text { (promoter) }\end{array}$ & $\begin{array}{l}\mathrm{C} / \mathrm{C} \\
\mathrm{T} / \mathrm{C} \\
\mathrm{T} / \mathrm{T}\end{array}$ & $\begin{array}{c}147(50.0) \\
122(41.5) \\
25(8.5)\end{array}$ & $\begin{array}{c}246(55.7) \\
168(38.0) \\
28(6.3)\end{array}$ & $0.82(0.65-1.04)$ & 0.098 & $0.80(0.59-1.07)$ & 0.130 & $0.73(0.42-1.27)$ & 0.270 \\
\hline $\begin{array}{l}\text { rs2455084 } \\
\text { (intron 1) }\end{array}$ & $\begin{array}{l}\mathrm{T} / \mathrm{T} \\
\mathrm{A} / \mathrm{T} \\
\mathrm{A} / \mathrm{A}\end{array}$ & $\begin{array}{c}184(61.3) \\
96(32.0) \\
20(6.7)\end{array}$ & $\begin{array}{r}252(53.5) \\
172(36.5) \\
47(10.0)\end{array}$ & $1.31(1.04-1.65)$ & 0.020 & $1.38(1.03-1.85)$ & 0.032 & $1.55(0.90-2.68)$ & 0.110 \\
\hline $\begin{array}{l}\text { rs1393009 } \\
\text { (intron 1) }\end{array}$ & $\begin{array}{l}\mathrm{T} / \mathrm{T} \\
\mathrm{T} / \mathrm{C} \\
\mathrm{C} / \mathrm{C}\end{array}$ & $\begin{array}{c}183(62.9) \\
89(30.6) \\
19(6.5)\end{array}$ & $\begin{array}{r}252(53.4) \\
173(36.6) \\
47(10.0)\end{array}$ & $1.37(1.09-1.73)$ & 0.007 & $1.48(1.10-1.99)$ & 0.010 & $1.58(0.91-2.76)$ & 0.096 \\
\hline $\begin{array}{l}\text { rs7539485 } \\
\text { (intron 2) }\end{array}$ & $\begin{array}{l}\mathrm{C} / \mathrm{C} \\
\mathrm{T} / \mathrm{C} \\
\mathrm{T} / \mathrm{T}\end{array}$ & $\begin{array}{c}183(61.8) \\
92(31.1) \\
21(7.1)\end{array}$ & $\begin{array}{r}239(54.6) \\
155(35.4) \\
44(10.1)\end{array}$ & $1.28(1.01-1.61)$ & 0.037 & $1.35(1.00-1.82)$ & 0.051 & $1.46(0.85-2.51)$ & 0.160 \\
\hline $\begin{array}{l}\text { rs4660616 } \\
\text { (intron 3) }\end{array}$ & $\begin{array}{l}\mathrm{T} / \mathrm{T} \\
\mathrm{T} / \mathrm{G} \\
\mathrm{G} / \mathrm{G}\end{array}$ & $\begin{array}{r}182(60.1) \\
100(33) \\
21(6.9)\end{array}$ & $\begin{array}{r}251(53.3) \\
171(36.3) \\
49(10.4)\end{array}$ & $1.28(1.02-1.60)$ & 0.033 & $1.32(0.98-1.77)$ & 0.063 & $1.56(0.92-2.66)$ & 0.095 \\
\hline $\begin{array}{l}\text { rs510157 } \\
\text { (intron 4) }\end{array}$ & $\begin{array}{l}\mathrm{G} / \mathrm{G} \\
\mathrm{T} / \mathrm{G} \\
\mathrm{T} / \mathrm{T}\end{array}$ & $\begin{array}{c}184(62.0) \\
93(31.3) \\
20(6.7)\end{array}$ & $\begin{array}{r}239(53.5) \\
163(36.5) \\
45(10.1)\end{array}$ & $1.33(1.06-1.68)$ & 0.015 & $1.42(1.05-1.91)$ & 0.022 & $1.55(0.90-2.68)$ & 0.110 \\
\hline $\begin{array}{l}\text { rs343386 } \\
\text { (intron 4) }\end{array}$ & $\begin{array}{l}\mathrm{T} / \mathrm{T} \\
\mathrm{T} / \mathrm{C} \\
\mathrm{C} / \mathrm{C}\end{array}$ & $\begin{array}{c}182(60.3) \\
99(32.8) \\
21(7.0)\end{array}$ & $\begin{array}{r}249(53.1) \\
173(36.9) \\
47(10.0)\end{array}$ & $1.28(1.02-1.60)$ & 0.032 & $1.34(1.00-1.80)$ & 0.050 & $1.49(0.87-2.55)$ & 0.140 \\
\hline $\begin{array}{l}\text { rs12732892 } \\
\text { (intron 4) }\end{array}$ & $\begin{array}{l}\mathrm{T} / \mathrm{T} \\
\mathrm{T} / \mathrm{C} \\
\mathrm{C} / \mathrm{C}\end{array}$ & $\begin{array}{c}217(72.6) \\
69(23.1) \\
13(4.3)\end{array}$ & $\begin{array}{c}320(68.1) \\
128(27.2) \\
22(4.7)\end{array}$ & $1.17(0.90-1.52)$ & 0.240 & $1.24(0.90-1.71)$ & 0.180 & $1.08(0.54-2.18)$ & 0.830 \\
\hline $\begin{array}{l}\text { rs343389 } \\
\text { (intron 4) }\end{array}$ & $\begin{array}{l}\mathrm{C} / \mathrm{C} \\
\mathrm{T} / \mathrm{C} \\
\mathrm{T} / \mathrm{T}\end{array}$ & $\begin{array}{c}183(61.0) \\
96(32.0) \\
21(7.0)\end{array}$ & $\begin{array}{r}252(53.5) \\
172(36.5) \\
47(10.0)\end{array}$ & $1.29(1.02-1.61)$ & 0.029 & $1.36(1.01-1.82)$ & 0.040 & $1.47(0.86-2.52)$ & 0.150 \\
\hline $\begin{array}{l}\text { rs343376 } \\
\text { (exon 5, } \\
\text { Ala161 Val) }\end{array}$ & $\begin{array}{l}\mathrm{C} / \mathrm{C} \\
\mathrm{T} / \mathrm{C} \\
\mathrm{T} / \mathrm{T}\end{array}$ & $\begin{array}{c}219(72.5) \\
70(23.2) \\
13(4.3)\end{array}$ & $\begin{array}{c}321(68.3) \\
126(26.8) \\
23(4.9)\end{array}$ & $1.16(0.90-1.51)$ & 0.250 & $1.22(0.89-1.68)$ & 0.210 & $1.14(0.57-2.29)$ & 0.700 \\
\hline $\begin{array}{l}\text { rs585320 } \\
\text { (intron 7) }\end{array}$ & $\begin{array}{l}\mathrm{T} / \mathrm{T} \\
\mathrm{T} / \mathrm{C} \\
\mathrm{C} / \mathrm{C}\end{array}$ & $\begin{array}{c}217(72.1) \\
69(22.9) \\
15(5.0)\end{array}$ & $\begin{array}{c}320(67.7) \\
130(27.5) \\
23(4.9)\end{array}$ & $1.14(0.88-1.48)$ & 0.300 & $1.24(0.90-1.70)$ & 0.190 & $0.97(0.50-1.90)$ & 0.940 \\
\hline
\end{tabular}

P-values were obtained from logistic regression analyses with the log-additive, dominant and recessive models controlling age and gender as covariates. Bold numbers indicate significant associations. FOXJ3, forkhead box J3; SNP, single nucleotide polymorphism; RA, rheumatoid arthritis; n, number of subjects; OR, odds ratio; CI, confidence interval.

Table IV: Haplotype association of FOXJ3 in patients with rheumatoid arthritis and control subjects.

\begin{tabular}{|c|c|c|c|c|c|c|c|c|c|}
\hline \multirow[b]{2}{*}{ Haplotype } & \multirow[b]{2}{*}{ Genotype } & \multirow{2}{*}{$\frac{\mathrm{RA}}{\mathrm{n}(\%)}$} & \multirow{2}{*}{$\frac{\text { Control }}{\mathrm{n}(\%)}$} & \multicolumn{2}{|c|}{ Log-additive } & \multicolumn{2}{|c|}{ Dominant } & \multicolumn{2}{|c|}{ Recessive } \\
\hline & & & & OR $(95 \% \mathrm{CI})$ & P-value & OR $(95 \% \mathrm{CI})$ & $\mathrm{P}$-value & OR $(95 \% \mathrm{CI})$ & P-value \\
\hline \multirow[t]{3}{*}{ TCCTTGTCTTC } & $\mathrm{H} / \mathrm{H}$ & $77(25.1)$ & $105(22.1)$ & $1.00(0.82-1.22)$ & 0.995 & $0.86(0.62-1.18)$ & 0.350 & $1.18(0.84-1.66)$ & 0.329 \\
\hline & $\mathrm{H} /-$ & $140(45.6)$ & $246(51.7)$ & & & & & & \\
\hline & $-/-$ & $90(29.3)$ & $125(26.3)$ & & & & & & \\
\hline \multirow[t]{3}{*}{ TCCTTGTCTTT } & $\mathrm{H} / \mathrm{H}$ & $25(8.1)$ & $28(5.9)$ & $1.28(1.02-1.61)$ & 0.035 & $1.35(1.01-1.80)$ & 0.043 & $1.42(0.81-2.48)$ & 0.221 \\
\hline & $\mathrm{H} /-$ & $122(39.7)$ & $165(34.7)$ & & & & & & \\
\hline & $-/-$ & $160(52.1)$ & $283(59.5)$ & & & & & & \\
\hline \multirow[t]{3}{*}{ CTTCCTGTCAC } & $\mathrm{H} / \mathrm{H}$ & $13(4.2)$ & $22(4.6)$ & $0.86(0.67-1.12)$ & 0.264 & $0.82(0.60-1.12)$ & 0.212 & $0.91(0.45-1.84)$ & 0.798 \\
\hline & $\mathrm{H} /-$ & $74(24.1)$ & $133(27.9)$ & & & & & & \\
\hline & $-/-$ & $220(71.7)$ & $321(67.4)$ & & & & & & \\
\hline \multirow[t]{3}{*}{ TCTTCTGTCAC } & $\mathrm{H} / \mathrm{H}$ & $3(1.0)$ & $4(0.8)$ & $0.66(0.45-0.96)$ & 0.032 & $0.60(0.40-0.91)$ & 0.017 & $1.16(0.26-5.24)$ & 0.843 \\
\hline & $\mathrm{H} /-$ & $34(11.1)$ & $84(17.6)$ & & & & & & \\
\hline & $-/-$ & $270(87.9)$ & $388(81.5)$ & & & & & & \\
\hline
\end{tabular}

P-values were obtained from logistic regression analyses with the log-additive, dominant and recessive models. Bold numbers indicate significant associations. FOXJ3, forkhead box J3; RA, rheumatoid arthritis; n, number of subjects; OR, odds ratio; CI, confidence interval. 
Table V. Genotype frequencies of FOXJ3 polymorphisms in rheumatoid arthritis patients with anti-CCP (negative) and anti-CCP (positive).

\begin{tabular}{|c|c|c|c|c|c|c|c|c|c|}
\hline \multirow{2}{*}{$\frac{\mathrm{SNP}}{\text { (Locus) }}$} & \multirow[b]{2}{*}{ Genotype } & \multirow{2}{*}{$\frac{\text { anti-CCP }(-)}{n(\%)}$} & \multirow{2}{*}{$\frac{\operatorname{anti-CCP}(+)}{n(\%)}$} & \multicolumn{2}{|c|}{ Log-additive } & \multicolumn{2}{|c|}{ Dominant } & \multicolumn{2}{|c|}{ Recessive } \\
\hline & & & & OR $(95 \% \mathrm{CI})$ & P-value & OR $(95 \% \mathrm{CI})$ & P-value & OR $(95 \% \mathrm{CI})$ & P-value \\
\hline $\begin{array}{l}\text { rs2282404 } \\
\text { (promoter) }\end{array}$ & $\begin{array}{l}\mathrm{C} / \mathrm{C} \\
\mathrm{T} / \mathrm{C} \\
\mathrm{T} / \mathrm{T}\end{array}$ & $\begin{array}{r}36(43.4) \\
38(45.8) \\
9(10.8)\end{array}$ & $\begin{array}{c}43(48.3) \\
37(41.6) \\
9(10.1)\end{array}$ & $0.89(0.56-1.40)$ & 0.60 & $0.83(0.45-1.52)$ & 0.54 & $0.94(0.35-2.50)$ & 0.89 \\
\hline $\begin{array}{l}\text { rs2455084 } \\
\text { (intron 1) }\end{array}$ & $\begin{array}{l}\mathrm{T} / \mathrm{T} \\
\mathrm{A} / \mathrm{T} \\
\mathrm{A} / \mathrm{A}\end{array}$ & $\begin{array}{r}45(52.3) \\
33(38.4) \\
8(9.3)\end{array}$ & $\begin{array}{c}58(64.4) \\
28(31.1) \\
4(4.4)\end{array}$ & $0.64(0.39-1.05)$ & 0.08 & $0.62(0.33-1.14)$ & 0.12 & $0.44(0.13-1.53)$ & 0.18 \\
\hline $\begin{array}{l}\text { rs1393009 } \\
\text { (intron 1) }\end{array}$ & $\begin{array}{l}\mathrm{T} / \mathrm{T} \\
\mathrm{T} / \mathrm{C} \\
\mathrm{C} / \mathrm{C}\end{array}$ & $\begin{array}{r}44(55.0) \\
29(36.2) \\
7(8.8)\end{array}$ & $\begin{array}{c}58(64.4) \\
28(31.1) \\
4(4.4)\end{array}$ & $0.69(0.42-1.14)$ & 0.15 & $0.68(0.36-1.26)$ & 0.22 & $0.48(0.13-1.70)$ & 0.24 \\
\hline $\begin{array}{l}\text { rs } 7539485 \\
\text { (intron 2) }\end{array}$ & $\begin{array}{l}\mathrm{C} / \mathrm{C} \\
\mathrm{T} / \mathrm{C} \\
\mathrm{T} / \mathrm{T}\end{array}$ & $\begin{array}{r}44(54.3) \\
29(35.8) \\
8(9.9)\end{array}$ & $\begin{array}{c}58(65.2) \\
27(30.3) \\
4(4.5)\end{array}$ & $0.66(0.40-1.09)$ & 0.10 & $0.65(0.35-1.21)$ & 0.17 & $0.42(0.12-1.46)$ & 0.16 \\
\hline $\begin{array}{l}\text { rs4660616 } \\
\text { (intron 3) }\end{array}$ & $\begin{array}{l}\mathrm{T} / \mathrm{T} \\
\mathrm{T} / \mathrm{G} \\
\mathrm{G} / \mathrm{G}\end{array}$ & $\begin{array}{r}44(51.2) \\
34(39.5) \\
8(9.3)\end{array}$ & $\begin{array}{c}58(64.4) \\
28(31.1) \\
4(4.4)\end{array}$ & $0.63(0.38-1.03)$ & 0.06 & $0.59(0.32-1.09)$ & 0.100 & $0.44(0.13-1.52)$ & 0.18 \\
\hline $\begin{array}{l}\text { rs510157 } \\
\text { (intron 4) }\end{array}$ & $\begin{array}{l}\mathrm{G} / \mathrm{G} \\
\mathrm{T} / \mathrm{G} \\
\mathrm{T} / \mathrm{T}\end{array}$ & $\begin{array}{r}45(55.6) \\
28(34.6) \\
8(9.9)\end{array}$ & $\begin{array}{c}58(65.2) \\
27(30.3) \\
4(4.5)\end{array}$ & $0.68(0.41-1.11)$ & 0.12 & $0.67(0.36-1.24)$ & 0.20 & $0.42(0.12-1.47)$ & 0.16 \\
\hline $\begin{array}{l}\text { rs343386 } \\
\text { (intron 4) }\end{array}$ & $\begin{array}{l}\mathrm{T} / \mathrm{T} \\
\mathrm{T} / \mathrm{C} \\
\mathrm{C} / \mathrm{C}\end{array}$ & $\begin{array}{r}45(52.3) \\
33(38.4) \\
8(9.3)\end{array}$ & $\begin{array}{c}58(64.4) \\
28(31.1) \\
4(4.4)\end{array}$ & $0.64(0.39-1.05)$ & 0.08 & $0.62(0.33-1.13)$ & 0.12 & $0.44(0.13-1.53)$ & 0.18 \\
\hline $\begin{array}{l}\text { rs12732892 } \\
\text { (intron 4) }\end{array}$ & $\begin{array}{l}\mathrm{T} / \mathrm{T} \\
\mathrm{T} / \mathrm{C} \\
\mathrm{C} / \mathrm{C}\end{array}$ & $\begin{array}{r}58(69.0) \\
20(23.8) \\
6(7.1)\end{array}$ & $\begin{array}{c}66(73.3) \\
23(25.6) \\
1(1.1)\end{array}$ & $0.70(0.40-1.21)$ & 0.20 & $0.81(0.42-1.57)$ & 0.53 & $0.13(0.02-1.12)$ & 0.03 \\
\hline $\begin{array}{l}\text { rs343389 } \\
\text { (intron 4) }\end{array}$ & $\begin{array}{l}\mathrm{C} / \mathrm{C} \\
\mathrm{T} / \mathrm{C} \\
\mathrm{T} / \mathrm{T}\end{array}$ & $\begin{array}{r}45(54.2) \\
30(36.1) \\
8(9.6)\end{array}$ & $\begin{array}{c}58(64.4) \\
28(31.1) \\
4(4.4)\end{array}$ & $0.67(0.41-1.09)$ & 0.11 & $0.66(0.36-1.22)$ & 0.18 & $0.43(0.12-1.48)$ & 0.17 \\
\hline $\begin{array}{l}\text { rs343376 } \\
\text { (exon 5, } \\
\text { Ala161 Val) }\end{array}$ & $\begin{array}{l}\mathrm{C} / \mathrm{C} \\
\mathrm{T} / \mathrm{C} \\
\mathrm{T} / \mathrm{T}\end{array}$ & $\begin{array}{r}58(69.0) \\
20(23.8) \\
6(7.1)\end{array}$ & $\begin{array}{c}66(74.2) \\
22(24.7) \\
1(1.1)\end{array}$ & $0.67(0.38-1.17)$ & 0.16 & $0.76(0.39-1.49)$ & 0.43 & $0.13(0.02-1.14)$ & 0.03 \\
\hline $\begin{array}{l}\text { rs585320 } \\
\text { (intron 7) }\end{array}$ & $\begin{array}{l}\mathrm{T} / \mathrm{T} \\
\mathrm{T} / \mathrm{C} \\
\mathrm{C} / \mathrm{C}\end{array}$ & $\begin{array}{r}58(67.4) \\
21(24.4) \\
7(8.1)\end{array}$ & $\begin{array}{c}66(73.3) \\
23(25.6) \\
1(1.1)\end{array}$ & $0.65(0.38-1.12)$ & 0.12 & $0.75(0.39-1.44)$ & 0.39 & $0.12(0.01-0.99)$ & 0.015 \\
\hline
\end{tabular}

P-values were obtained from logistic regression analyses with the log-additive, dominant and recessive models controlling age and gender as covariates. Bold numbers indicate significant associations. FOXJ3, forkhead box J3; anti-CCP, anti-cyclic citrullinated peptide; SNP, single nucleotide polymorphism; n, number of subjects; OR, odds ratio; $\mathrm{CI}$, confidence interval.

adjustment for age and gender. The genotype distributions of FOXJ3 gene polymorphisms in the RA and control groups are shown in Table III. Of the 11 SNPs, seven (rs2455084, rs1393009, rs7539485, rs4660616, rs510157, rs343386 and rs343389) were significantly associated with the risk of RA. The rare alleles of rs2455084 (rare allele, A), rs1393009 (rare allele, C), rs7539485 (rare allele, T), rs4660616 (rare allele, G), rs510157 (rare allele, T), rs343386 (rare allele, C) and rs343389 (rare allele, T) were associated with a decreased risk of RA. The rest of the SNPs (rs2282404, rs12732892, rs343376 and rs585320) were not associated with RA (Table III).

FOXJ3, located on chromosome 1p34.2, consists of 622 amino acids and has a molecular mass of 68,928 Da. The missense polymorphism rs343376 (Ala162 Val) is the only SNP with heterozygosity $>0.1$ among the coding SNPs located in the FOXJ3 gene region (http://www.ncbi.nlm.nih.gov/SNP). CC, CT and TT genotype frequencies are 0.467, 0.417 and 0.117 in European; 0.822, 0.178 and 0.000 in Chinese; 0.750, 0.227 and 0.023 in Japanese and $0.467,0.433$ and 0.100 in Sub-Saharan African populations (http://www.ncbi.nlm.nih.
gov/SNP). The CC, CT and TT genotype frequencies in the population of the present study were $0.680,0.270$ and 0.040 , which are similar to those in the Japanese population. However, the rs 343376 polymorphism was not associated with RA in the study population (Table III). The SNP genotype frequencies obtained from the study population are similar to frequencies observed in the Japanese population, according to the NCBI website.

A strong LD block, including all SNPs, was constructed using the Gabriel method (Fig. 1B). Four haplotypes in the block exhibited frequencies $>0.05$ and all were used for haplotype association analysis. The frequency of the TCCTTGTCTTC haplotype was 0.465 , TCCTTGTCTTT 0.271 , CTTCCTGTCAC 0.175 and TCTTCTGTCAC 0.086 , respectively. Two haplotypes (TCCTTGTCTTT and TCTTCTGTCAC) were significantly associated with RA (Table IV).

To determine whether FOXJ3 SNPs influence the clinical features of RA, the RA group was divided according to clinical characteristics such as ESR ( $\geq 30$ or $<30 \mathrm{~mm} / \mathrm{h}$ ), CRP $(\geq 0.5$ or 
$<0.5 \mathrm{mg} / \mathrm{dl}$ ), bone erosion (positive or not), anti-CCP (positive and negative) and RF (positive or negative). We observed an association between anti-CCP and SNP (rs585320). The SNP rs585320 was significant in the recessive model $(\mathrm{OR}=0.12$, 95\% CI=0.01-0.99, P-value=0.015; Table V). However, no association was observed between FOXJ3 polymorphisms and the clinical characteristics (ESR, CRP, bone erosion and RF).

\section{Discussion}

RA is a common autoimmune disease with a complex etiology involving genetic and environmental factors. The association between FOXJ3 polymorphisms and susceptibility to RA was investigated in the present study. The results suggested that certain SNPs in the FOXJ3 gene contribute to RA. The case-control analysis demonstrated that of 11 SNPs analyzed in FOXJ3, seven (rs2455084, rs1393009, rs7539485, rs4660616, rs510157, rs343386 and rs343389) are significantly associated with a susceptibility to RA. Moreover, two haplotypes (TCCTTGTCTTT and TCTTCTGTCAC) are significantly associated with RA. To the best of our knowledge, this is the first study to demonstrate that FOXJ3 polymorphisms are associated with RA.

It was also analyzed whether FOXJ3 polymorphisms are related to diagnostic markers reflecting RA disease activity, such as the levels of CRP, ESR, anti-CCP and RF, and the presence of bone erosion. Based on a value for ESR of $30 \mathrm{~mm} / \mathrm{h}, \mathrm{CRP}$ of $0.5 \mathrm{mg} / \mathrm{dl}$, the existence of bone erosion and the presence of anti-CCP (positive or negative) and RF (positive and negative); the RA patient sample was divided into two clinical subgroups and the correlation between clinical data and FOXJ3 polymorphisms was investigated. The results demonstrated that anti-CCP was associated with FOXJ3 SNP.

Forkhead proteins are a large family of functionally diverse transcription factors that have been implicated in a variety of cellular processes. Recently, several studies have demonstrated that this family is involved in the regulation of immune responses. Nik Tavakoli et al (24) reported that FOXP3 is critical in the regulation and development of $\mathrm{T}_{\text {reg }}$ cells. FOXN1 modulates the thymic stroma and regulates thymopoiesis, and FOXOs are also involved in the maintainance of quiescence and tolerance of $\mathrm{T}$ and B cells (25). Moreover, it has been suggested that FOXJ1 was associated with autoimmune diseases, such as allergic rhinitis and systemic lupus erythematosus $(20,21)$. However, studies concerning the function of FOXJ3 remain to be conducted. Thus far it has only been suggested that FOXJ3 may be involved in the development of skeletal muscle, and the peripheral and central nervous system (17). Therefore, further studies are required to investigate the function of FOXJ3 (rs585320).

In the present study, seven significant SNPs were located in introns and did not result in an amino acid change. According to the dbSNP database (http://www.ncbi.nlm.nih.gov/SNP/) and the HapMap database (http://www.hapmap.org/), seven SNPs in exons of the FOXJ3 gene have been identified; however, with the exception of rs343376, these SNPs remain unknown or $<0.1$ about heterozygosity of them. Since low heterozygosity means little genetic variability, these SNPs were excluded from the present study and only one coding SNP (rs343376) was analyzed. This SNP (rs343376) was not associated with RA in the study population. It was suggested that significant polymorphisms in introns may be markers rather than direct contributors to genetic functions. The effects of other genetic variations linked to these polymorphisms may exhibit functional significance. However, there remains a possibility that these intronic polymorphisms are involved in its genetic function via the change resulting from alternative splicing (26).

In conclusion, the results of the present study suggest that FOXJ3 polymorphisms are associated with susceptibility to RA in the study population. To the best of our knowledge, this study is the first to analyze the association between FOXJ3 SNPs and RA. Moreover, the polymorphism/association information identified in this study may be useful for further genetic studies of RA.

\section{Acknowledgements}

This work was supported by the Priority Research Centers Program through the National Research Foundation of Korea (NRF) funded by the Ministry of Education, Science and Technology (2009-0093829) and by a grant from the Institute of Bio-Science and Technology (IBST) at Dankook University in 2010 .

\section{References}

1. Silman AJ and Pearson JE: Epidemiology and genetics of rheumatoid arthritis. Arthritis Res 4 (Suppl 3): S265-S272, 2002.

2. Firestein GS: Evolving concepts of rheumatoid arthritis. Nature 423: 356-361, 2003.

3. Klareskog L, Padyukov L, Rönnelid J and Alfredsson L: Genes, environment and immunity in the development of rheumatoid arthritis. Curr Opin Immunol 18: 650-655, 2006.

4. Hall FC, Weeks DE, Camilleri JP, et al: Influence of the HLA-DRB1 locus on susceptibility and severity in rheumatoid arthritis. QJM 89: 821-829, 1996.

5. Kong KF, Yeap SS, Chow SK and Phipps ME: HLA-DRB1 genes and susceptibility to rheumatoid arthritis in three ethnic groups from Malaysia. Autoimmunity 35: 235-239, 2002.

6. Mattey DL, Dawes PT, Clarke S, et al: Relationship among the HLA-DRB1 shared epitope, smoking, and rheumatoid factor production in rheumatoid arthritis. Arthritis Rheum 47: 403-407, 2002.

7. Begovich AB, Carlton VE, Honigberg LA, et al: A missense single-nucleotide polymorphism in a gene encoding a protein tyrosine phosphatase (PTPN22) is associated with rheumatoid arthritis. Am J Hum Genet 75: 330-337, 2004.

8. Karlson EW, Chibnik LB, Cui J, et al: Associations between human leukocyte antigen, PTPN22, CTLA4 genotypes and rheumatoid arthritis phenotypes of autoantibody status, age at diagnosis and erosions in a large cohort study. Ann Rheum Dis 67: 358-363, 2008.

9. Ikari K, Momohara S, Inoue E, et al: Haplotype analysis revealed no association between the PTPN22 gene and RA in a Japanese population. Rheumatology (Oxford) 45: 1345-1348, 2006.

10. Kawasaki E, Awata T, Ikegami H, et al: Systematic search for single nucleotide polymorphisms in a lymphoid tyrosine phosphatase gene (PTPN22): association between a promoter polymorphism and type 1 diabetes in Asian populations. Am J Med Genet A 140: 586-593, 2006.

11. Suzuki A, Yamada R, Chang X, et al: Functional haplotypes of PADI4, encoding citrullinating enzyme peptidylarginine deiminase 4 , are associated with rheumatoid arthritis. Nat Genet 34: 395-402, 2003.

12. Tokuhiro S, Yamada R, Chang X, et al: An intronic SNP in a RUNX1 binding site of SLC22A4, encoding an organic cation transporter, is associated with rheumatoid arthritis. Nat Genet 35: 341-348, 2003.

13. Kochi Y, Yamada R, Suzuki A, et al: A functional variant in FCRL3, encoding Fc receptor-like 3, is associated with rheumatoid arthritis and several autoimmunities. Nat Genet 37: 478-485, 2005. 
14. Plenge RM, Padyukov L, Remmers EF, et al: Replication of putative candidate-gene associations with rheumatoid arthritis in $>4,000$ samples from North America and Sweden: association of susceptibility with PTPN22, CTLA4, and PADI4. Am J Hum Genet 77: 1044-1060, 2005.

15. Barton A, Bowes J, Eyre S, et al: A functional haplotype of the PADI4 gene associated with rheumatoid arthritis in a Japanese population is not associated in a United Kingdom population. Arthritis Rheum 50: 1117-1121, 2004.

16. Carlsson P and Mahlapuu M: Forkhead transcription factors: key players in development and metabolism. Dev Biol 250: 1-23, 2002

17. Landgren $\mathrm{H}$ and Carlsson P: FoxJ3, a novel mammalian forkhead gene expressed in neuroectoderm, neural crest, and myotome. Dev Dyn 231: 396-401, 2004.

18. Kaestner KH, Knochel W and Martinez DE: Unified nomenclature for the winged helix/forkhead transcription factors. Genes Dev 14: 142-146, 2000.

19. Dejaco C, Duftner C, Grubeck-Loebenstein B and Schirmer M: Imbalance of regulatory $\mathrm{T}$ cells in human autoimmune diseases. Immunology 117: 289-300, 2006.
20. Li CS, Chae SC, Lee JH, et al: Identification of single nucleotide polymorphisms in FOXJ1 and their association with allergic rhinitis. J Hum Genet 51: 292-297, 2006.

21. Li CS, Zhang Q, Lim MK, et al: Association of FOXJ1 polymorphisms with systemic lupus erythematosus and rheumatoid arthritis in Korean population. Exp Mol Med 39: 805-811, 2007.

22. Arnett FC, Edworthy SM, Bloch DA, et al: The American Rheumatism Association 1987 revised criteria for the classification of rheumatoid arthritis. Arthritis Rheum 31: 315-324, 1988.

23. Lewis CM: Genetic association studies: design, analysis and interpretation. Brief Bioinform 3: 146-153, 2002.

24. Nik Tavakoli N, Hambly BD, Sullivan DR and Bao S: Forkhead box protein 3: essential immune regulatory role. Int $\mathrm{J}$ Biochem Cell Biol 40: 2369-2373, 2008.

25. Coffer PJ and Burgering BM: Forkhead-box transcription factors and their role in the immune system. Nat Rev Immunol 4: 889-899, 2004

26. Ast G: How did alternative splicing evolve? Nat Rev Genet 5: 773-782, 2004 